\title{
THE MULTIPLICITY OF SHAPES IN TI NUCLEI
}

\author{
L.L. RIEDINGER, W. REVIOL, J.M. LEWIS, W.F. MUELLER, B.H. SMITH, \\ and J.-Y. ZHANG \\ Department of Physics, University of Tennessee, \\ Knoxville, TN 37996-1200, USA
}

S.M. FISCHER, M.P. CARPENTER, R.V.F. JANSSENS, B. CROWELL, I. AHMAD, D.J. BLUMENTHAL, T.L. KHOO, T. LAURITSEN, and D. NISIUS

Physics Division, Argonne National Laboratory, Argonne, IL 60439, USA

B. CEDERWALL

Nuclear Science Division, Lawrence Berkeley National Laboratory, Berkeley, CA 94720, USA

\begin{abstract}
The results of experiments on rotational bands in light $\mathrm{Tl}$ nuclei are used to indicate the richness of shape phenomena in the vicinity of a closed proton shell. Rotational bands associated with normal prolate and oblate shapes as well as with a superdeformed shape are observed.
\end{abstract}

\section{Introduction}

Thallium nuclei $(Z=81)$ provide a rich laboratory for the study of nuclear shapes. In spite of the location adjacent to a closed shell number of protons $(\mathrm{Pb}-Z=82)$, $\mathrm{Tl}$ nuclei exhibit an impressive array of excitations built on different shapes, prolate and oblate, normal and superdeformed. The properties of these various modes of excitation present challenges to nuclear models and spur interest in understanding the configurations involved. Furthermore, the richness of structures built upon different shapes and different configurations gives a good opportunity in future measurements to understand new questions that have arisen, e.g. the conditions under which discrete lines might be observed for direct one-step decay from superdeformed to low-lying bands of normal deformation.

This paper describes some of the recent measurements on rotational bands associated with normal and superdeformed shapes in $\mathrm{Tl}$ isotopes. We have been involved in a series of experiments which lead to the assignment of rotational bands in the following nuclei:

- Superdeformed bands in ${ }^{191} \mathrm{Tl},{ }^{1}{ }^{192} \mathrm{Tl}^{2,3}$ and perhaps ${ }^{189} \mathrm{Tl}$ from recent

a Present Address: Manne Siegbahn Institute, Stockholm, Sweden 
GAMMASPHERE measurements; these bands are calculated to have a deformation of $\beta_{2} \sim 0.45$ according to the calculations of Satula et al.";

- Oblate normal deformed bands built on the $\pi h_{9 / 2}$ excitation in ${ }^{187} \mathrm{Tl}{ }^{5}$ (see also the work of Lane et al. ${ }^{6}$ ), ${ }^{189} \mathrm{Tl}^{7}$, and ${ }^{191} \mathrm{Tl}{ }^{7,8}$ and on the $\pi i_{13 / 2}$ excitation in ${ }^{191} \mathrm{Tl}$; these bands are representative of a deformation $\beta_{2}=$ $0.15-0.20, \gamma=-60^{\circ}$;

- Prolate bands of large deformation built on the $\pi h_{9 / 2}$ and $\pi i_{13 / 2}$ intruder states, seen in ${ }^{187} \mathrm{Tl}{ }^{5,6}$ and in ${ }^{189} \mathrm{Tl}^{7}$; most of these are described by $\beta_{2}$ $\sim 0.25$, although the $\pi h_{9 / 2}$ band in ${ }^{189} \mathrm{Tl}$ has properties best described by a smaller prolate deformation, $\beta_{2} \approx 0.15$;

- Oblate bands in odd-odd ${ }^{192} \mathrm{Tl}$.

In this paper some of the most important aspects of these various cases are presented and compared with calculations. Possibilities for future measurements are discussed.

\section{Bands of Normal Deformation in ${ }^{189} \mathrm{TI}$}

For a study of normal and superdeformed bands in ${ }^{189} \mathrm{Tl}$, we collected $2.8 \cdot 10^{8}$ triple and higher fold coincidences with Early Implementation GAMMASPHERE? These data have enabled us to extend the previously known ${ }^{9} 9 / 2[505]$ and $1 / 2[660]$ bands and to establish three new bands. The proposed level scheme for the normal deformed states in ${ }^{189} \mathrm{Tl}$ is shown in Fig. 1 (new bands are labeled $1 a, 1 b$, and 5). Spin and parity assignments to energy levels are based on previous decay work and on our analysis of DCO ratios. Some of these assignments are tentative and given in parentheses. This scheme does not show the complete information on levels built on the $I^{\pi}=13 / 2^{+}$state, but only the strongest cascade.

The lowest lying band, built upon the $9 / 2[505]$ isomer $\left(t_{1 / 2}=1.4 \mathrm{~m}\right)$ and labeled 1 , is associated with an oblate deformed shape $\left(\beta_{2} \sim 0.15\right)$. This band is crossed by another strongly-coupled band (1a) establishing a sharp $\left(\nu i_{13 / 2}\right)^{2}$ backbend. In this crossing region, another band of one signature (1b) feeds in and is most likely due to the oblate $1 / 2$ [550] (hole) configuration? The decoupled band 4 is based on the prolate $1 / 2[660]$ intruder orbital $\left(\beta_{2} \sim 0.27\right)$. The structure on the left in the figure contains decoupled band 5 with $I^{\pi} \geq$ $\left(9 / 2^{-}\right)$and is proposed to be based on the prolate $1 / 2[541]$ intruder orbital. The decay out of this band proceeds mainly through a branch that feeds the known ${ }^{10} I^{\pi}=3 / 2^{+}$state, as indicated by coincidence relationships involving 


\section{DISCLAIMER}

This report was prepared as an account of work sponsored by an agency of the United States Government. Neither the United States Government nor any agency thereof, nor any of their employees, makes any warranty, express or implied, or assumes any legal liability or responsibility for the accuracy, completeness, or usefulness of any information, apparatus, product, or process disclosed, or represents that its use would not infringe privately owned rights. Reference herein to any specific commercial product, process, or service by trade name, trademark, manufacturer, or otherwise does not necessarily constitute or imply its endorsement, recommendation, or favoring by the United States Government or any agency thereof. The views and opinions of authors expressed herein do not necessarily state or reflect those of the United States Government or any agency thereof.

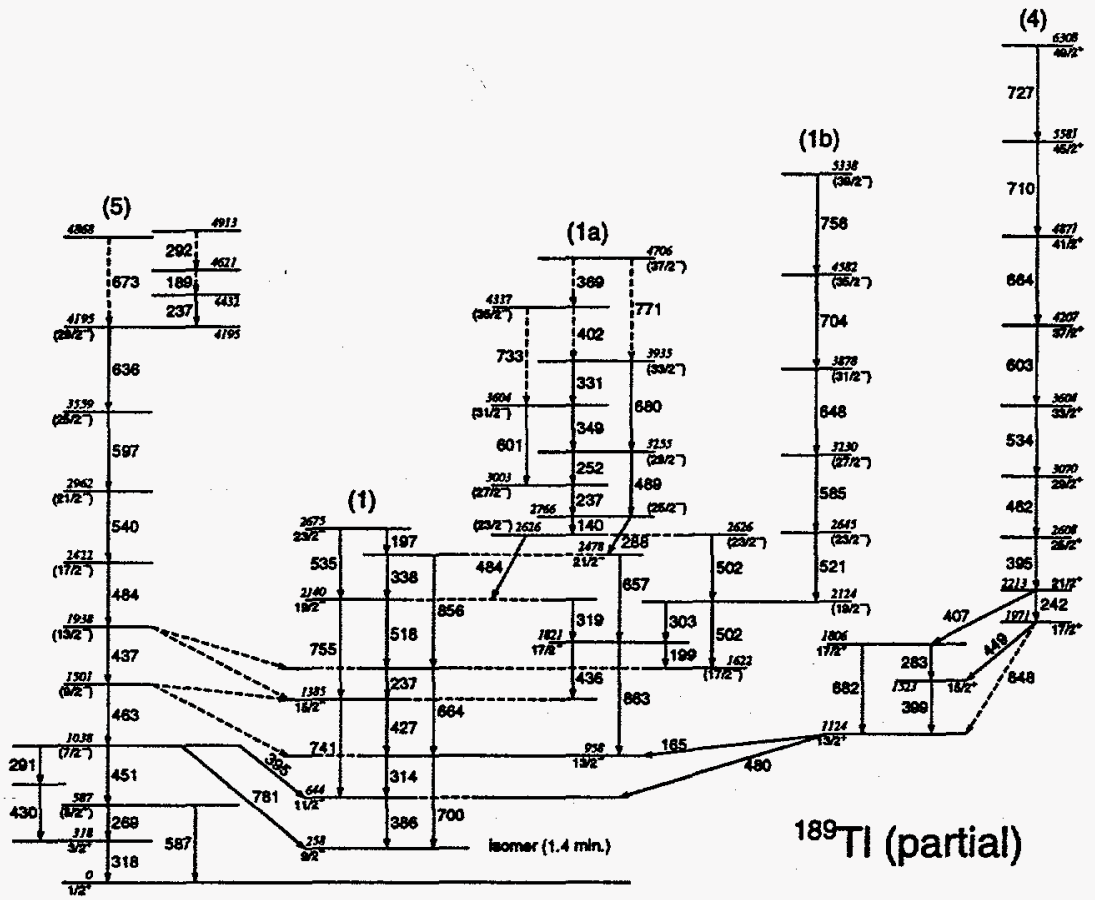

Figure 1: Partial level scheme of ${ }^{189} \mathrm{Tl}$ from high-spin spectroscopic studies using the ${ }^{156} \mathrm{Gd}\left({ }^{37} \mathrm{Cl}, 4 \mathrm{n}\right)$ reaction. The band structures viewed as oblate and prolate bands are shown. Levels are labeled by the proposed spins, parities, and excitation energies (assuming the state fed by the $318 \mathrm{keV} 3 / 2^{+} \rightarrow 1 / 2^{+}$transition $^{10}$ is the ground state).

a $318 \mathrm{keV}$ transition, and bypasses the $9 / 2$ [505] isomer. This $318 \mathrm{keV}$ line is assumed to be the known $3 / 2^{+} \rightarrow 1 / 2^{+}$ground state transition, which determines all level energies in the scheme. Such a decay pathway for highspin states is a rather unique observation made possible by the resolving power of a new-generation $\gamma$-ray detector array.

A band of strong prolate deformation $\left(\beta_{2} \sim 0.26\right)$ intrudes into the low energy spectrum of slightly oblate $\mathrm{Hg}(Z=80)$ nuclei, and comes to successively lower excitation energies down to $N=102^{182} \mathrm{Hg}^{11}$ Dracoulis et al. ${ }^{12}$ have measured this band in ${ }^{180} \mathrm{Hg}$, and find that this prolate excitation starts up in energy compared to ${ }^{182} \mathrm{Hg}$. In $\mathrm{Pb}(Z=82)$ the pattern is less well established. Heese et al. ${ }^{13}$ and Baxter et al.$^{14}$ have recently established this prolate band in $N=104$ and $106{ }^{186,188} \mathrm{~Pb}$, and find that it is lower in energy in the former.

It is a sensitive test of nuclear models to predict the deformation and the 
energetics of this prolate intruder band, not only in isotopes of $\mathrm{Hg}$ and $\mathrm{Pb}$, but also in intermediate $\mathrm{Tl}$ as the high- $j$ proton configurations couple to it. Band 4 in ${ }^{189} \mathrm{Tl}$ (see Fig. 1) corresponds to this prolate intruder configuration, and it comes to lower excitation energies in ${ }^{187} \mathrm{Tl}^{5,6}$ and in ${ }^{185} \mathrm{Tl}^{6}$ The quasiparticle alignment in this decoupled band is high ( $\sim 6 \hbar$ initially), as one would expect for the $1 / 2[660]$ configuration. We have performed calculations based upon the Nilsson potential and find that the deformation of this $\pi i_{13 / 2}$ band in ${ }^{187} \mathrm{Tl}$ and ${ }^{189} \mathrm{Tl}$ is similar to that in the $\mathrm{Hg}$ isotone, $\beta_{2} \approx 0.26$. Calculations based upon a Woods-Saxon potential reach a similar conclusion. 6

There is also a $\pi h_{9 / 2}$ band coupled to this prolate minimum in ${ }^{185} \mathrm{Tl}^{6}$ and in ${ }^{187} \mathrm{Tl}^{5,6}$ However, the situation is different in ${ }^{189} \mathrm{Tl}$. Band 5 (see Fig. 1) is likely $\pi h_{9 / 2}$ in nature, and resembles bands in ${ }^{185} \mathrm{Tl}$ and ${ }^{187} \mathrm{Tl}$ in the way it decays to the yrast $\pi h_{9 / 2}$ structure (oblate). However; in the case of ${ }^{189} \mathrm{Tl}$, band 5 has lower alignment and its strongest decay mode proceeds to the low lying states built on the ground state. Rather than an initial quasiparticle alignment of $\sim 4 \hbar$ as in the lighter $\mathrm{Tl}$ isotopes or in ${ }^{185} \mathrm{Au},{ }^{15}$ band 5 in ${ }^{189} \mathrm{Tl}$ has only $1 \hbar$. Our calculations ${ }^{5}$ explain this as being due to the presence of a prolate minimum of intermediate deformation, $\beta_{2} \approx 0.15$. Whereas three protons are placed in the low- $K h_{9 / 2}$ orbitals for the well deformed band in the lighter $\mathrm{Tl}$ nuclei, the occupation for this excitation in ${ }^{189} \mathrm{Tl}$ is only one, producing a smaller prolate deformation. This again indicates the richness of shape effects in $\mathrm{Tl}$.

\section{Superdeformed Bands in odd-A Tl}

The region of superdeformation around $A=190$ extends from $\mathrm{Hg}(Z=80)$ to Po (84) and from $N=108$ or 109 to 114 . This region is distinguished by similar moments of inertia (increasing gradually as a function of rotational frequency) and transitions within the second well down to rather low spin ( $I$ as low as $\sim 6 \hbar$ ). In order to determine the orbitals responsible for the formation of these extended nuclear shapes, it is important to map this superdeformed region. We have performed experiments at the low $N$ edge of this region, finding two superdeformed bands in ${ }^{191} \mathrm{Tl}^{1}$ and searching for bands in ${ }^{189} \mathrm{Tl}$. And, we have discovered superdeformed bands in ${ }^{192} \mathrm{Tl}$ using the Argonne-Notre Dame array $^{2}$ and GAMMASPHERE. ${ }^{3}$

An intensive search for superdeformed bands has been made on our ${ }^{189} \mathrm{Tl}$ data obtained from the Early Implementation GAMMASPHERE. This analysis has revealed a weak signal of seven transitions in ${ }^{189} \mathrm{Tl}(367,409,448,488$, 527,565 , and $603 \mathrm{keV}$ ) with an average spacing of about $40 \mathrm{keV}$ and estimated intensities in the order of $0.1 \%$ of the yield of the $4 \mathrm{n}$ reaction channel. Due to 
the weakness of these intensities and the presence of a rather high background (mainly due to fission), it is difficult to determine the coincidence relationships between all these transitions to firmly establish the presence of a band. The features of this weak signal fit to characteristics of other superdeformed bands in the region in that the $\mathcal{J}^{(2)}(\hbar \omega)$ moments of this possible band in ${ }^{189} \mathrm{Tl}$ are increasing smoothly and with a slope similar to those observed for the sets of bands in the ${ }^{191,193} \mathrm{Tl}$ isotopes based on the 5/2[642] excitation. ${ }^{1,16}$ However, a candidate for a signature partner band in the ${ }^{189} \mathrm{Tl}$ data is not found. It is expected that a superdeformed band in ${ }^{189} \mathrm{Tl}$ would be populated very weakly, considering the trend of SD band intensities in the isotopic chain of ${ }^{189-192} \mathrm{Hg}$ (see e.g. Drigert et al. ${ }^{17}$ ) together with the fact that SD bands are more weakly populated in $\mathrm{Tl}$ nuclei than in their $\mathrm{Hg}$ isotones.

The deformation of the nucleus in the superdeformed minimum is a quantity that is calculable in various theoretical models, and which is therefore a test of our understanding of the nuclear dynamics in the second well. One model ${ }^{4}$ has led to deformations calculated as a function of $\beta_{2}$ and $\beta_{4}$ for light $\mathrm{Tl}$ nuclei, as shown in Fig. 2. The trends in the neighboring $\mathrm{Hg}$ and $\mathrm{Pb}$ nuclei are the same. The predicted $\beta_{2}$ gradually decreases for the same configuration $\left(\pi 6^{5} \nu 7^{2}\right)$ as $N$ decreases, with the total change being roughly $10 \%$ from ${ }^{193} \mathrm{Tl}$ to ${ }^{189} \mathrm{Tl}$. The question is how this can be measured. Transition quadrupole moments in superdeformed bands have been measured in a few cases in the $A=190$ region, but these are difficult experiments with uncertainties that could mask the effect shown in Fig. 2. While not as direct of an indication of the deformation, there are other ways to assess the change in deformation.

Two superdeformed bands have been observed in ${ }^{195} \mathrm{Tl}_{,}^{18,19,20}{ }_{193} \mathrm{Tl}^{16,21}$ and ${ }^{191} \mathrm{Tl}{ }^{1}$ In each case these bands are assumed to be the signature partners expected for the $\pi 6^{5} \nu 7^{2}$ configuration, i.e. $5 / 2[642]$, and in ${ }^{193} \mathrm{Tl}$ and ${ }^{195} \mathrm{Tl}$ there are observed transitions linking the signature partners. Our data on ${ }^{189} \mathrm{Tl}$ are not good enough to be sure of the assignment of the one superdeformed band. Nevertheless, we include this one suggested band in the plot of moments of inertia vs. the square of the rotational frequency in Fig. 3. Note that the $\mathcal{J}^{(2)}$ moment is slightly larger for the heavier $\mathrm{Tl}$ isotopes, and the question is whether this is in line with the deformation trend predicted by Satula et al. ${ }^{4}$ and shown in Fig. 2. In order to extract an estimate of this $\mathcal{J}^{(2)}$ dependence on $N$, we plot the values as a function of $\omega^{2}$. The total angular momentum along the rotation axis can be written as

$$
I_{x}=i+\omega\left(\mathcal{J}_{0}+\mathcal{J}_{1} \omega^{2}\right)
$$

and thus

$$
\mathcal{J}^{(2)}=d I_{x} / d \omega=\mathcal{J}_{0}+3 \mathcal{J}_{1} \omega^{2}
$$

where $\mathcal{J}_{0}$ and $\mathcal{J}_{1}$ are the normal Harris parameters describing a rotating ref- 


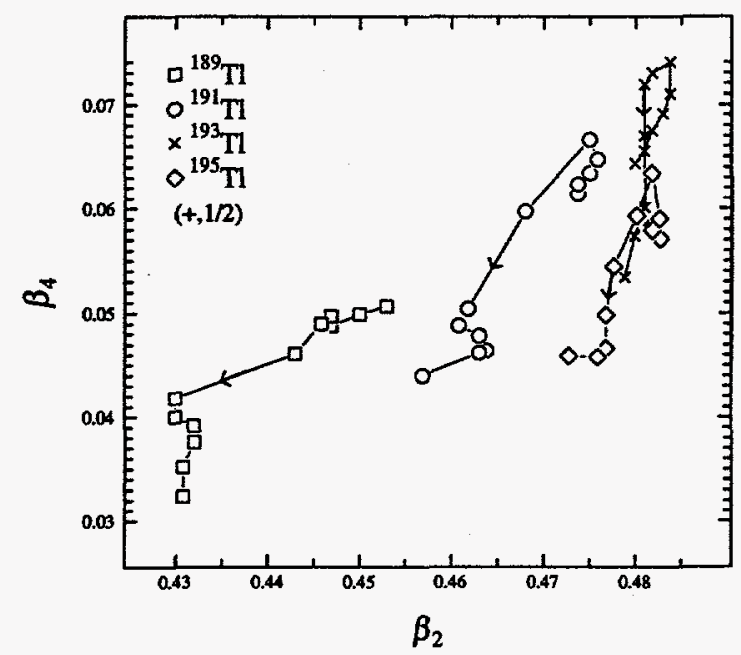

Figure 2: Calculated $\beta_{2}$ and $\beta_{4}$ deformations for superdeformed bands in four $T 1$ nuclei. The figure is reproduced from the paper by Satula et al. ${ }^{4}$ Arrows indicate the evolution of the nuclear shape with increasing $\hbar \omega$.

erence. In this way, a linear fit to the plots in Fig. 3 can lead to the extraction of the Harris parameters. While $\mathcal{J}_{1}$ is related to the variation of the second moment with frequency, the $\mathcal{J}_{0}$ moment is proportional to the deformation $\beta_{2}{ }^{22}$ As shown in Fig. 3, a line through the ${ }^{193} \mathrm{Tl} \mathcal{J}^{(2)}$ values gives a $\mathcal{J}_{0}$ of approximately $95 \hbar^{2} / \mathrm{MeV}$, while that for ${ }^{189} \mathrm{Tl}$ yields about $90 \hbar^{2} / \mathrm{MeV}$. This $5 \%$ decrease in $\mathcal{J}_{0}$ likely indicates a drop in $\beta_{2}$, which is about half of that predicted by Satula et al. ${ }^{4}$ Thus, the apparent reduction in deformation seen in the odd- $A \mathrm{Tl}$ isotopes follows the trend suggested earlier by Drigert et al. ${ }^{17}$ from a similar analysis of the lighter $\mathrm{Hg}$ isotopes $(A=189-192)$. This small decrease in $\mathcal{J}^{(2)}$ makes it important to be sure of our preliminary assignment in ${ }^{189} \mathrm{Tl}$, and suggests a new experiment at GAMMASPHERE.

\section{Superdeformed Bands in ${ }^{192} \mathrm{Tl}$}

The majority of superdeformed sequences near $A=190$ shows the characteristic steady increase of $\mathcal{J}^{(2)}$ as a function of $\hbar \omega$. It is generally accepted that this effect is mainly caused by gradually (and successively) aligning $N=7\left(\nu j_{15 / 2}\right)$ and $N=6\left(\pi i_{13 / 2}\right)$ quasiparticles. Superdeformation studies in odd-odd nuclei, particularly ${ }^{192} \mathrm{Tl}^{2,3}$ have been performed as "blocking experiments" to test this alignment picture. We find ${ }^{3}$ four superdeformed sequences in ${ }^{192} \mathrm{Tl}$, 


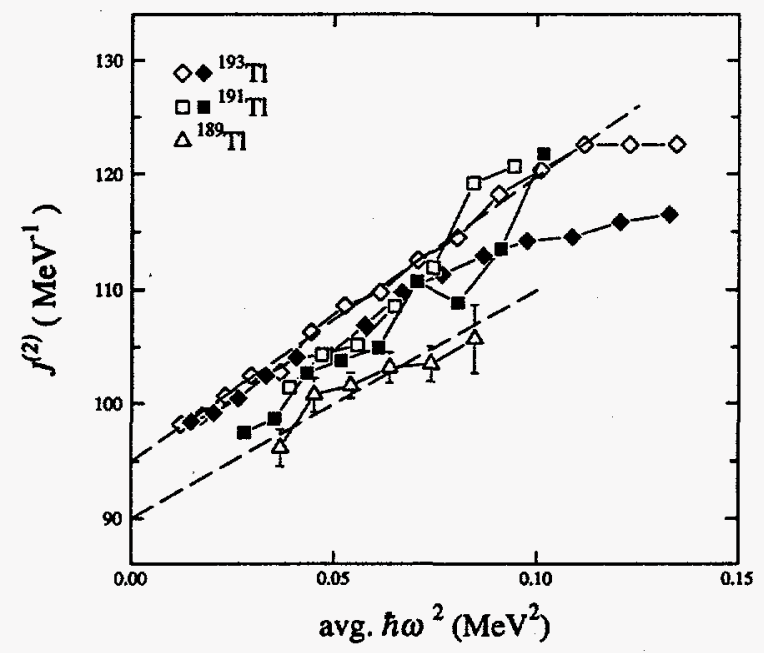

Figure 3: Moments of inertia $\left(\mathcal{J}^{(2)}\right)$ vs. rotational frequency squared for known superdeformed bands in ${ }^{193} \mathrm{Tl}_{4}^{16,21}{ }^{191} \mathrm{Tl}_{4}^{1}$ and ${ }^{189} \mathrm{Tl}$ (our recent results). The data for ${ }^{189} \mathrm{Tl}$ are tentative (see text for details).

two with an increasing $\mathcal{J}^{(2)}$ and the other two with a non-characteristic behavior of essentially constant $\mathcal{J}^{(2)}$ values. The latter two sequences are viewed as signature partners. By comparison with calculations, it is shown ${ }^{2,3}$ that the $\nu j_{15 / 2}$ alignment (calculated to occur in ${ }^{191} \mathrm{Tl}$ and ${ }^{190,192} \mathrm{Hg}$ ) and the $\pi i_{13 / 2}$ alignment (calculated to occur in ${ }^{191} \mathrm{Hg}$ and ${ }^{190,192} \mathrm{Hg}$ ) are blocked. Thus, a $\pi 6^{5} \nu 7^{3}$ assignment is proposed for the two ${ }^{192} \mathrm{Tl}$ sequences with approximately constant $\mathcal{J}^{(2)}(\hbar \omega)$ values. The data on ${ }^{192} \mathrm{Tl}$ thus provide evidence that alignments in the two high- $N$ orbitals are responsible for the steady rise in $\mathcal{J}^{(2)}$ seen in neighboring superdeformed nuclei.

\section{Normal Deformed Bands in ${ }^{192} \mathrm{Tl}$}

The GAMMASPHERE data taken for the study of superdeformed bands in ${ }^{192} \mathrm{Tl}$ have also been used to study in detail the yrast and near-yrast states of this nucleus. We have built a new level scheme ${ }^{24}$ shown together with the superdeformed bands in Fig. 4. The proposed spin and parity assignments to the normally deformed states are preliminary. To make these assignments more concrete, we have begun an analysis of the multipolarities of the $\gamma$-ray transitions by evaluating DCO (Directional Correlations from Oriented states) ratios. 
The low-spin part of this level scheme is adopted from previous work by Kreiner et al. ${ }^{25}$ Although a link between the $8^{-}$state and the lower lying $K^{x}=8^{-}$isomeric state $\left(t_{1 / 2}=272 \mathrm{~ns}\right)$ is not observed in the data, it is evident that this isomer is populated strongly as indicated by the presence of a $250.6-\mathrm{keV} \gamma$ ray in coincidence with most transitions. Therefore, the lower limit for the $K$ value of the observed levels is $6-8 \hbar$ and the proposed spin assignments can be viewed as reasonable. The known strongly coupled negative parity band ${ }^{25}$ (band 1 in Fig. 4) has been extended. This band is observed to be crossed by a new strongly-coupled band $1 a$, which extends the level scheme up to a spin of $24 \hbar$ and an excitation energy of $\sim 5.2 \mathrm{MeV}$. The band structures labeled 2 and 3 are entirely new observations, each consisting of two signature partner sequences. The upper part of band 2 is linked to band 1 by relatively high-energy $\gamma$-rays with DCO ratios that are consistent with $E 1$ multipolarity. This part of band 2 is linked to the bandhead sequence by a $135 \mathrm{keV} \gamma$ ray, which has been tentatively assigned as an $I \rightarrow I$ transition. Band 3 decays to lower lying states which are located in the crossing region of bands 1 and $1 a$. There is evidence in the data for one more new band structure which cannot be related to the scheme of strongly-coupled bands shown in Fig. 4, nor can it be assigned to a neighboring nucleus. It is likely that these $\gamma$-ray transitions represent a decoupled band in ${ }^{192} \mathrm{Tl}$.

For preliminary configuration assignments to bands $1,1 \mathrm{a}$, and 2 , we assume that the odd-proton occupies an $h_{9 / 2}$ orbital, in analogy to states in odd- $A \mathrm{Tl}$ neighboring nuclei above the $9 / 2^{-}$isomer. This proton orbital is known to have a large influence on the shape of $A \sim 190$ nuclei which typically minimize in total energy at slightly oblate deformations with $\beta_{2} \sim 0.15$. To identify the active neutron orbital involved, we compare the experimental routhian energies as a function of rotational frequency to those for yrast bands in the neighboring ${ }^{191,192} \mathrm{Hg}$ nuclei. ${ }^{26}$ While in ${ }^{192} \mathrm{Hg}$ the $A B$ neutron pair undergoes a rotational alignment at a crossing frequency of $0.21 \mathrm{MeV}$, this crossing is blocked in ${ }^{191} \mathrm{Hg}$ by the odd neutron and instead a delayed $B C$ crossing occurs at $\hbar \omega_{c} \sim 0.27 \mathrm{MeV}$. Very similar to the ${ }^{191} \mathrm{Hg}$ case is the crossing behavior of bands 1 and $1 \mathrm{a}$ in ${ }^{192} \mathrm{Tl}$ with $\hbar \omega_{c} \sim 0.26 \mathrm{MeV}$ and a similar alignment gain $(\Delta i \sim 9 \hbar)$. Thus, band 1 is proposed to be based on the $\pi 9 / 2[505] \otimes \nu 5 / 2[642]\left(K=7^{-}\right)$configuration and band 1a on the corresponding four-quasiparticle configuration $\left(K=7^{-}\right) \otimes\left(i_{13 / 2}\right)^{2}$. These assignments agree with the earlier interpretation for band $1 .^{25}$ The $e^{\prime}(\hbar \omega)$ plot for band 2 resembles the ${ }^{192} \mathrm{Hg}$ case, thereby excluding the excitation of a single $i_{13 / 2}$ neutron. Since band 2 interacts with band 1 , it must also be high- $K$ in nature. Most likely, band 2 is based on a $\pi 9 / 2[505] \otimes \nu 5 / 2[503]$ configuration with $K=7^{+}$. This would be also the energetically favorable coupling of parallel 
proton and neutron spins according to the Gallagher-Moszkowski rule. ${ }^{27}$ The two-quasiparticle configuration assignments for bands 1 and 2 are supported by an analysis of $B(M 1) / B(E 2)$ ratios. A comparison between experimental $B(M 1) / B(E 2)$ ratios and predictions obtained from the semi-classical model by Dönau and Frauendorf ${ }^{28,29}$ indicates agreement between data and model for the $K=7$ couplings.

\section{Considerations about the Decay-out of the Superdeformed Bands}

An exciting recent development in superdeformation studies has been the observation of discrete one-step decays from superdeformed bands in ${ }^{194} \mathrm{Hg}$ to several low-lying normal states. ${ }^{30}$ These transitions seem to be $E 1$ in nature, which is not surprising in view of the statistical character of the superdeformed decay process. The power of the new arrays now opens up the possibility of searching for these direct decays from superdeformed states in other nuclei. One key question is whether the $K$ quantum number plays a role in the occurrence of these one-step decays. It is logical for superdeformed bands to have a discrete value of $K$, since they are composed of quasiparticle orbitals with well defined $K$. However, decay out of superdeformed bands is thought to occur at a spin where there is sufficient mixing with a host of close-lying normal deformed states. ${ }^{31}$ Due to the density of the latter type of states at these excitation energies, it is unlikely that there is preservation of a discrete value of $K$ for the normal states with which the superdeformed bands mix at the decay-out point. Nevertheless, one wonders if the mixing still picks out in the normal state a piece of the wave function with $K$ similar to that of the superdeformed state. In this way, it is perhaps possible that $K$ would be important in the selection of the routes for direct decay to low-lying normal states.

There has been another recent discussion of the unexpected preservation of the $K$ quantum number among high-lying states, i.e. those populated in the neutron capture process. Rekstad et al ${ }^{32}$ studied the intensities of primary $\gamma$ ray transitions in ${ }^{168} \mathrm{Er}$ and ${ }^{168} \mathrm{Hf}$ (and more recently in ${ }^{166} \mathrm{Ho}^{33}$ ) to low-lying states of known values of $K$. They have concluded that the capture states have to be assigned certain values of $K$ in order to explain the observed distribution of $\gamma$-ray decay to the low lying states. By assigning appropriate $K$ values to these capture states, they found that decay to states which are forbidden by the normal $K$-selection rules was significantly reduced compared to that to the allowed $K$ states at low energy. This has been a controversial suggestion, and has started a debate about whether those neutron-capture data are being correctly interpreted. ${ }^{34}$ The same question needs to be investigated for the 


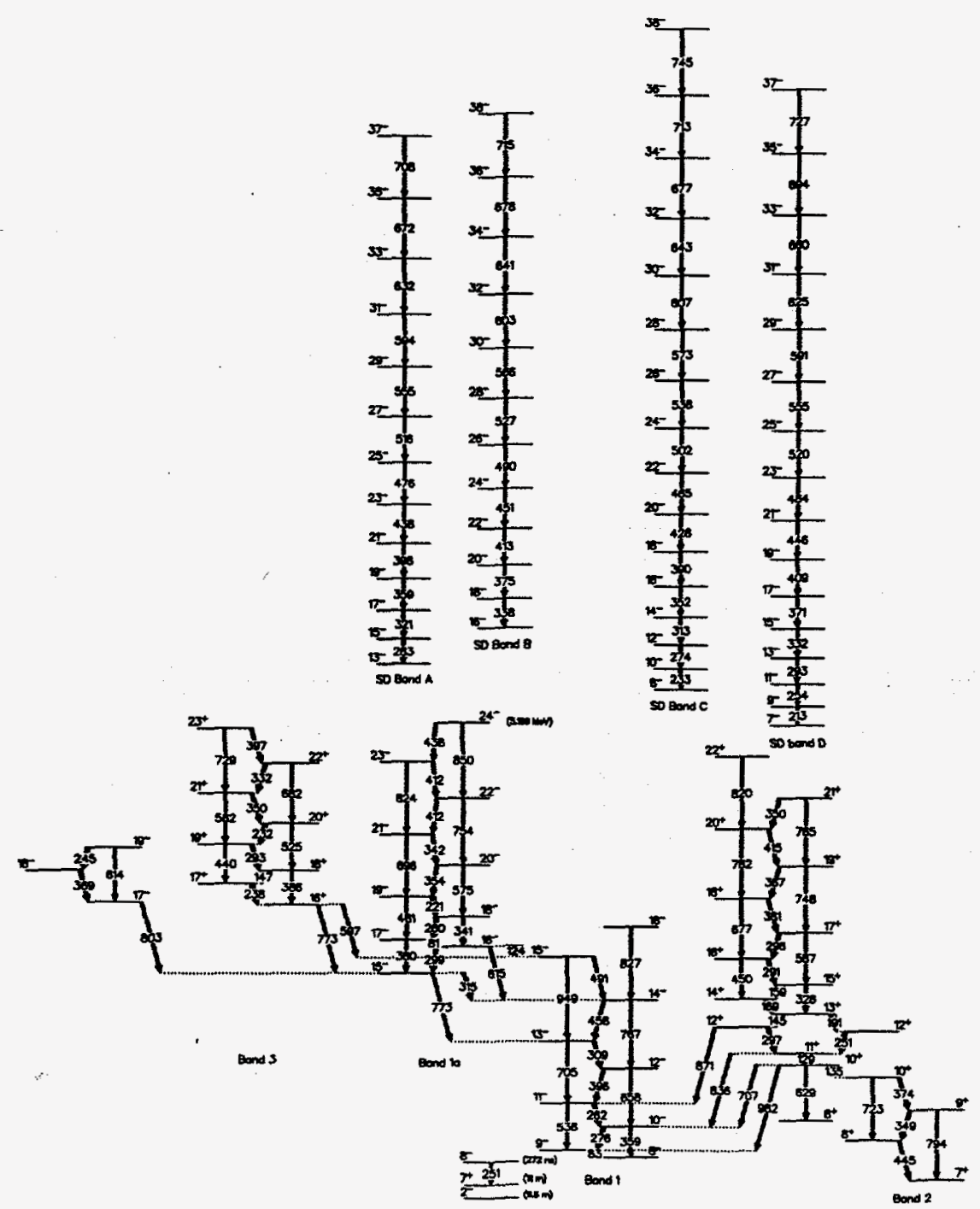

Figure 4: Preliminary level scheme for ${ }^{192} \mathrm{Tl}$ from our recent work. The low lying $7^{+}$and $8^{-}$states are isomeric with half lives of $11 \mathrm{~m}$ and $272 \mathrm{~ns}$, respectively. ${ }^{25}$ For the levels above these states, spin and parity assignments are tentative. Also included are the four observed superdeformed bands, with suggested spins according to the analysis of Fischer et al. ${ }^{3}$ 
states lying near the superdeformed bands at the decay-out point.

To test this idea, we propose to look more closely in the future at odd-odd ${ }^{192} \mathrm{Tl}$. As discussed by Fischer et al., ${ }^{3}$ bands $\mathrm{A}$ and $\mathrm{B}$ have the doubly blocked crossing, and thus should be $\pi i_{13 / 2} \nu j_{15 / 2}$ in nature, i.e. $5 / 2[642]_{\pi} 3 / 2[761]_{\nu}$ giving $K^{\pi}=4^{-}$. If the $K$ quantum number is important in the direct one-step decays, then there should be no such transitions from superdeformed bands $A$ and $\mathrm{B}$ to the $K=7$ bands at low energy. Bands $\mathrm{C}$ and $\mathrm{D}$ involve the same proton state, coupled to neutron orbital 9/2[624] (giving $K^{x}=7^{+}$) or 5/2[512] $\left(5^{-}\right)$. If the former assignment is proper, then there would be no $K$ hindrance in the the one-step decays to the low lying $K=7$ bands. In addition, the preference for $E 1$ transitions from the superdeformed structures would further lead to a selection of direct decays to Band $1\left(K^{\pi}=7^{-}\right)$, but not to Band 2 $\left(7^{+}\right)$. The standard fits of the superdeformed energies to a rotational formula yield the spins for bands $C$ and D proposed in the level scheme (see Fig. 4). The lowest levels of Bands $\mathrm{C}$ and $\mathrm{D}$ should thus directly decay to the $I=7,8$ states of Band 1, but not to Band 2, if the $K^{\pi}=7^{+}$assignment is correct. These decay lines were not seen in the our recent GAMMASPHERE measurement, but an experiment with a fuller complement of detectors could be successful in searching for such decays.

\section{Conclusions}

The rich variety of rotational phenomena in $\mathrm{Tl}$ nuclei is impressive, especially considering that the number of protons is one short of a closed shell. We have demonstrated here the existence of rotational structures built on configurations associated with both prolate and oblate shapes, normal and superdeformed. Calculations of the shapes as a function of configuration are generally successful in providing a basis for explaining the observations. There are unanswered questions that can only be addressed by future measurements. For example, it is important to test the predicted trend ${ }^{4}$ that the deformation in the second well should decrease by $10 \%$ for light $\mathrm{Tl}$ nuclei. Our preliminary result on one superdeformed band in ${ }^{189} \mathrm{Tl}$ indicates a moment of inertia that is compatible with this prediction, but the absence of the expected signature partner leaves the status in question. The recent observation ${ }^{30}$ of one-step decay from superdeformed bands in ${ }^{194} \mathrm{Hg}$ to low lying levels raises the question of whether selection rules based on the change in the $K$ quantum number are important for these transitions. Among the questions concerning states of normal deformation, the issue of whether the prolate minimum in $\mathrm{Tl}$ continues to drop in energy for ${ }^{181} \mathrm{Tl}$ and ${ }^{183} \mathrm{Tl}$, and perhaps comes even lower than the oblate minimum is an important one. The completion of GAMMASPHERE should allow 
experiments to search for answers to each of these questions and will presumably yield even more fascinating results.

\section{Acknowledgments}

The authors wish to acknowledge experimental colleagues at Lawrence Berkeley National Laboratory (I.Y. Lee, A.O. Macchiavelli), Oak Ridge National Laboratory (C. Baktash, J.D. Garrett, N.R. Johnson, and F.K. McGowan), and formerly at the University of Tennessee (S. Pilotte and C.-H. Yu). Discussions on theoretical topics with W. Nazarewicz have been very helpful. This work has been sponsored by the Department of Energy, Nuclear Physics Division, under contract DE-FG05-87ER40361, W-31-109-ENG-38 and DEAC03-76SF00098.

\section{References}

1. S. Pilotte et al., Phys. Rev. C 49, 718 (1994).

2. Y. Liang et al., Phys. Rev. C 46, R2136 (1992).

3. S.M. Fischer et al., Phys. Rev. C, in press (1996).

4. W. Satula et al, Nucl. Phys. A 529, 289 (1991).

5. W. Reviol et al., Phys. Rev. C 49, R587 (1994).

6. G.J. Lane et al., Nucl. Phys. A 586, 316 (1995).

7. W. Reviol et al., Phys. Scr. T56, 167 (1995).

8. J.M. Lewis, Masters Thesis, Univ. Tenn. 1994.

9. M.-G. Porquet et al., Phys. Rev. C 44, 2445 (1991).

10. E. Coenen et al., Phys. Rev. Lett. 54, 1783 (1985).

11. W.C. Ma et al., Phys. Lett. B 139, 276 (1984).

12. G.D. Dracoulis et al., Phys. Lett. B 208, 365 (1988).

13. J. Heese et al., Phys. Lett. B 302, 390 (1993).

14. A.M. Baxter et al., Phys. Rev. C 48, R2140 (1993).

15. A.J. Larabee et al., Phys. Lett. B 169, 21 (1986).

16. P.B. Fernandez et al., Nucl. Phys. A 517, 386 (1990).

17. M.W. Drigert et al., Nucl. Phys. A 530, 452 (1991).

18. F. Azaiez et al., Phys. Rev. Lett. 66, 1030 (1991).

19. J. Duprat et al., Conference on Physics from Large Gamma-ray Detector Arrays (report LBL-35687, Berkeley, 1994).

20. J. Duprat et al., Phys. Lett. B 341, 6 (1994).

21. S. Bouneau et al., Nucl. Phys. A, submitted (1996).

22. L.L. Riedinger, Phys. Scr. 24, 312 (1981).

23. F. Azaiez et al., Z. Phys. A 338, 471 (1991). 
24. B.H. Smith et al., Bull. Amer. Phys. Soc. 4016241995.

25. A.J. Kreiner et al., Phys. Rev. C 21, 933 (1980).

26. H.H. Hübel et al., Nucl. Phys. A 453, 316 (1986).

27. C. J. Gallagher and S. A. Moszkowski, Phys. Rev. 111, 1282 (1958).

28. F. Dönau and S. Frauendorf, in Conference on High Angular Momentum Properties of Nuclei ed. N.R. Johnson (Harwood, New York, 1992).

29. F. Dönau, Nucl. Phys. A 471, 469 (1987).

30. T.L. Khoo et al., Phys. Rev. Lett. in press 1996.

31. E. Vigezzi, R. A. Broglia, and T. Dossing, Phys. Lett. B 249, 163 (1990).

32. J. Rekstad et al., Phys. Rev. C 47, 2621 (1993).

33. L. Bergholt et al., Phys. Rev. C 50, 493 (1994).

34. B.B. Barrett et al., Phys. Rev. C 45, R1417 (1992). 